Radiologe 2021 · 61:977-978

https://doi.org/10.1007/s00117-021-00923-2

Angenommen: 15. September 2021

(c) Springer Medizin Verlag $\mathrm{GmbH}$, ein Teil von Springer Nature 2021

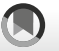

\title{
Strukturierte Befundung: warum und warum nicht jetzt?
}

\author{
Maximilian F. Reiser ${ }^{1}$. Peter Mildenberger ${ }^{2}$ \\ 'Klinik und Poliklinik für Radiologie, Klinikum der Universität München, München, Deutschland \\ ${ }^{2}$ Klinik und Poliklinik für Diagnostische und Interventionelle Radiologie, Universitätsmedizin Mainz, \\ Mainz, Deutschland
}

Liebe Leserinnen und Leser,

strukturierte Befundung oder "structured reporting" (SR) ist seit Langem ein Thema in der Radiologie. Unverändert wird jedoch ein Großteil der Befundung heute weiterhin als sog. Fließtext oder Freitext verfasst. Dies hat verschiedene Gründe: Die Radiologen trennen sich nur widerwillig von liebgewonnenen Gewohnheiten und langjährig eingeübten, bewährten Verfahren, zumal die bislang verfügbaren technischen Umsetzungen der SR meist mit einem erhöhten Aufwand verbunden sind und mehr Zeit beanspruchen, die den Radiologen oft fehlt.

Der Mehrwert einer strukturierten Befundung ist insbesondere für komplexe Befunde, beispielsweise in einem präoperativen Staging oder bei onkologischen Verlaufskontrollen, belegt, da die Befunde meist vollständiger sind und leichter vom Zuweiser aufgenommen werden können. Damit werden potenziell Fehler vermieden, und wesentliche kritische Befunde sind leichter zu erkennen. Der Vergleich mit der Labormedizin mag weit hergeholt erscheinen - ist es aber nicht. Stellen Sie sich einen Laborbefund in Fließtext vor, ohne farbliche Markierung der pathologischen Werte. Ein lautstarker Protest der Zuweiser wäre unvermeidbar, das Labor würde viele Zuweiser verlieren und die Idee würde sehr schnell verworfen.

In diesem Themenheft haben wir verschiedene Aspekte der strukturierten Befundung aufgegriffen und konnten dafür besonders kompetente Autoren gewinnen, bei denen wir uns sehr bedanken wollen.

Die deutschen, europäischen und internationalen radiologischen Fachgesellschaften unterstützen nachdrücklich die
SR. Ehe man sich aber über die Ausgestaltung und Funktionalität von SR-Systemen unterhält, muss klar sein, welche IT-Standards vorhanden sind und wie sie in SRSysteme integriert werden können. Entscheidend für die Akzeptanz der SR ist die nahtlose Integration in den Ablauf der Routinebefundung.

Große Hoffnungen und Erwartungen ruhen auf der künstlichen Intelligenz. Sie könnte wesentlich dazu beitragen, dass die SR praktisch handhabbar wird und einen substanziellen Mehrwert für alle Stakeholder erbringt - für die Radiologen, ihre klinischen Partner und nicht zuletzt, sondern zuvorderst für unsere Patientinnen und Patienten.

Der radiologische Befund kann damitzu einem objektiven Parameter werden, der auch für große Populationsstudien, ebenso wie für lokale Analysen nutzbar gemacht werden kann.

Noch in den 70er Jahren des letzten Jahrhunderts gab es in den radiologischen Abteilungen Tafeln mit maßstabsgetreuen Abbildungen von Kirschkernen, Kirschen, Äpfeln, Birnen, Eiern etc., um die Größe und Form pathologischer Veränderungen zu beschreiben. Aus heutiger Sicht ein naiver, ja anrührender Versuch, radiologische Befunde strukturiert zu kommunizieren.

Mit den zugegebenermaßen bisher ausbaufähigen Verfahren der SR eröffnet sich die Möglichkeit, mit quantitativen, konsensbasierten und reproduzierbaren Befunden einen noch wertvolleren Beitrag zur Versorgung der Patentinnen und Patienten zu leisten. Die Radiologinnen und Radiologen sollten, ja müssen diese Entwicklung aufmerksam beobachten, Lö- 


\section{Einführung zum Thema}

sungen von IT-Anbietern einfordern und umsetzen, sobald die Zeit gekommen ist.

Ihre

Maximilian Reiser

Peter Mildenberger

Korrespondenzadresse

Prof. em. Dr. Dr. h.c. Maximilian F. Reiser Klinik und Poliklinik für Radiologie, Klinikum der Universität München

Marchioninistraße 15, 81377 München,

Deutschland

Maximilian.Reiser@med.uni-muenchen.de

\section{Prof. Dr. Peter Mildenberger}

Klinik und Poliklinik für Diagnostische und Interventionelle Radiologie, Universitätsmedizin Mainz

Langenbeckstr. 1, 55131 Mainz, Deutschland mildenbe@uni-mainz.de

Interessenkonflikt. M.F. Reiser und P. Mildenberger geben an, dass kein Interessenkonflikt besteht.

\section{$\Sigma$}

\section{SpringerMedizin.de Lesen Sie Ihre Fachzeitschrift auch als ePaper!}

Als Abonnent können Sie Ihre Zeitschrift in verschiedenen Formaten lesen. Wählen Sie je nach Vorliebe und Situation aus, ob Sie die Zeitschrift als Print-Ausgabe, in Form von einzelnen Beiträgen auf springermedizin.de oder aber als komplette, elektronische ePaper-Ausgabe lesen möchten.

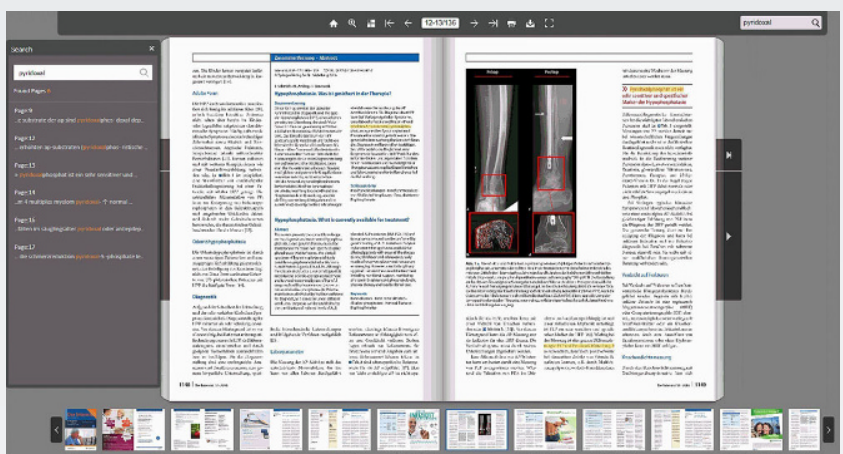

Die ePaper sind die identische Form der gedruckten Ausgaben. Sie sind nutzbar auf verschiedenen Endgeräten wie $\mathrm{PC}$, Tablet oder Smartphone

Das sind die Vorteile des ePapers:
$>$ Das verlinkte Inhaltsverzeichnis führt Sie direkt zum gewünschten Beitrag.
> Eine Suchfunktion ermöglicht das Auffinden von Schlagworten innerhalb der Zeitschrift.
$>$ Jede Ausgabe kann als PDF heruntergeladen und damit auch offline gelesen werden bzw. auch gespeichert oder ausgedruckt werden.
> Als Abonnent haben Sie Zugang zu allen ePaper-Ausgaben ab 2016.

Sie finden die ePaper auf SpringerMedizin.de bei der jeweiligen Ausgabe Ihrer Fachzeitschrift. Klicken Sie auf den Button „Ausgabe als ePaper lesen“. 